\title{
The Centre for Health Informatics at the University of New South Wales - a Clinical Informatics Research Centre
}

\author{
E. Coiera, F. Magrabi, V. Sintchenko, T. Zrimec, G. McDonnell, G. Chung, G. Tsafnat \\ Centre for Health Informatics, UNSW, Sydney, Australia
}

\begin{abstract}
Summary
Objectives: Building a sustainable health system in the 21stentury will require the reinvention of much of the present daysystem, and the intelligent use of information and communication technologies (IT) to deliver high quality, safe, efficient and affordable health care. The Centrefor Health Informatics (CH) is Australia's largest academic research group in this emerging discipline.

Methods: Our research is underpinned by a planning process, based ondifferentfuture scenariosfor the health system, which helps us identifylonger-term problems needing a sustained research effort. A research competency matrixis used to ensure that the Centrehas the requisite core capabilitiesin the research methods and tools needed to pussue our research program.

Resuls:s: The Centre's wookisinternationally recognized for its contributions in the development o fintelligent search systems to supportevidence-based healthcare, developing evaluation methodologies for IT, and i in understanding how communication shapes the safelyynd qualityofhealth caredelivery. Centre researchers also are working on safety models and standards forl IT in healthcare, mining complex genemicro array, medical literature and medical record data, building health system simulation methods to model the impact of health polizy changes, and developing novel computational methods to outomate the diagnosis of 3-D medical images.

Concusions: Anyindividual research group like CHI mustnecessarily focus on a few areas to allowitto develop sufficient research capacity to make novel and internationallysignificant contributions. As CHl approaches the end of itstirstdecade, itis becoming dear that developing capacaity becomesincreasingly challenging as the research territory changes under our feet, and that the Centre will continue to evolve and shiftitsfocus in the years to come.

\section{Keywords}

Academictraining, training programs, informatics, biomedical informatics

Geissbuhler A, Haux R, Kulikowski C, editiors. MIMA Yearbook of Medical Informatic 2007. Methods Inf Med 2007; 46Supp 1 : 141-8
\end{abstract}

\section{Introduction}

Post-genome the next healthcare revolution is in information and systems, or informatics. Building a sustainable health system in the 21st Century will require the reinvention of much of the present day system [1], and the intelligent use of information and communication technologies (ICT) to deliver high quality, safe, efficient and affordable health care.

The Centre for Health Informatics (CHI) is Australia's largest academic research group in this emerging discipline. Founded in 2000, CHI is specifically set up to foster cross-disciplinary research, and as such is a cross Faculty organization, bringing together academic researchers from the Faculties of Medicine and Engineering (mainly from the School of Computer Science and Engineering). The Centre is organizationally associated with the Faculty of Medicine.

The Centre's work is internationally recognized for its contributions in the development of intelligent search systems to support evidence-based healthcare, developing evaluation methodologies for ICT, and in understanding how communication shapes the safety and quality of health care delivery. Centre researchers also are working on safety models and standards for ICT in healthcare, mining complex gene micro array, medical literature and medical record data, building health system simulation methods to model the impact of health policy changes, and developing novel computational methods to automate the diagnosis of 3-D medical images.

The majority of staff at $\mathrm{CHI}$ are full time researchers, supported by competitive research grants and industry collaborations. We also have an active $\mathrm{PhD}$ and Masters by research program, taking in students from either a health or engineering background to work alongside Center staff on all of our research programs. In recent years students have completed research in areas such as clinical decision support, clinical communication processes, evaluation, information retrieval and intelligent image analysis. $\mathrm{CHI}$ also welcomes a small number of visiting International Fellows, who spend extend periods with the Centre working on shared research interests, and we have a regular stream of international students who come to us for shorter periods to complete research projects which contribute to the completion of a degree at their home institution.

\section{Four Futures for the Health Care System}

It may take a decade or more for today's research to successfully mature and have impact in the world, which means that any new research program needs to be 
solving problems that are somewhat in the future. Yet predicting the future is notoriously hard, and researchers are in a double bind in that they must both predict that future, and work back to identify problems for which present day research programs will offer a part solution. To assist in planning a research program that is most responsive to the near term needs of the health system, CHI's research program is underpinned by a scenario planning process, where we attempt to envisage different health system futures. In our model, we recognize that there are two major determinants of change - the stability of the environment within which the health system has to deliver its services, and the willingness or ability of the health system to adapt to these needs. Using these two broad forces, we have identified four broad scenarios, which now shape our thinking and research plans (Figure 1):

1. Making ICT Work: In this quadrant, faced with relatively stable operating conditions the health system can embark on incremental changes in response to projected future demands. A research program here would focus on assisting in the redesign of current health services using ICT. For example, working to understand why some ICT implementations succeed or fail, or helping shape the design of current software systems to improve their effectiveness would all fit within this research quadrant.

2. New Ways: Incremental changes to the health system may be insufficient to achieve the needed improvements in health service delivery in future years, given emerging demographic challenges such as clinical workforce shortages and the increasing burden of disease associated with an ageing population in most developed nations [1]. Consequently more radical models of care may need to be developed, evaluated and adopted. Rather than simply retrofitting the way things are done now with ICT to make them more 'efficient', work in this area seeks to radically reevaluate how things

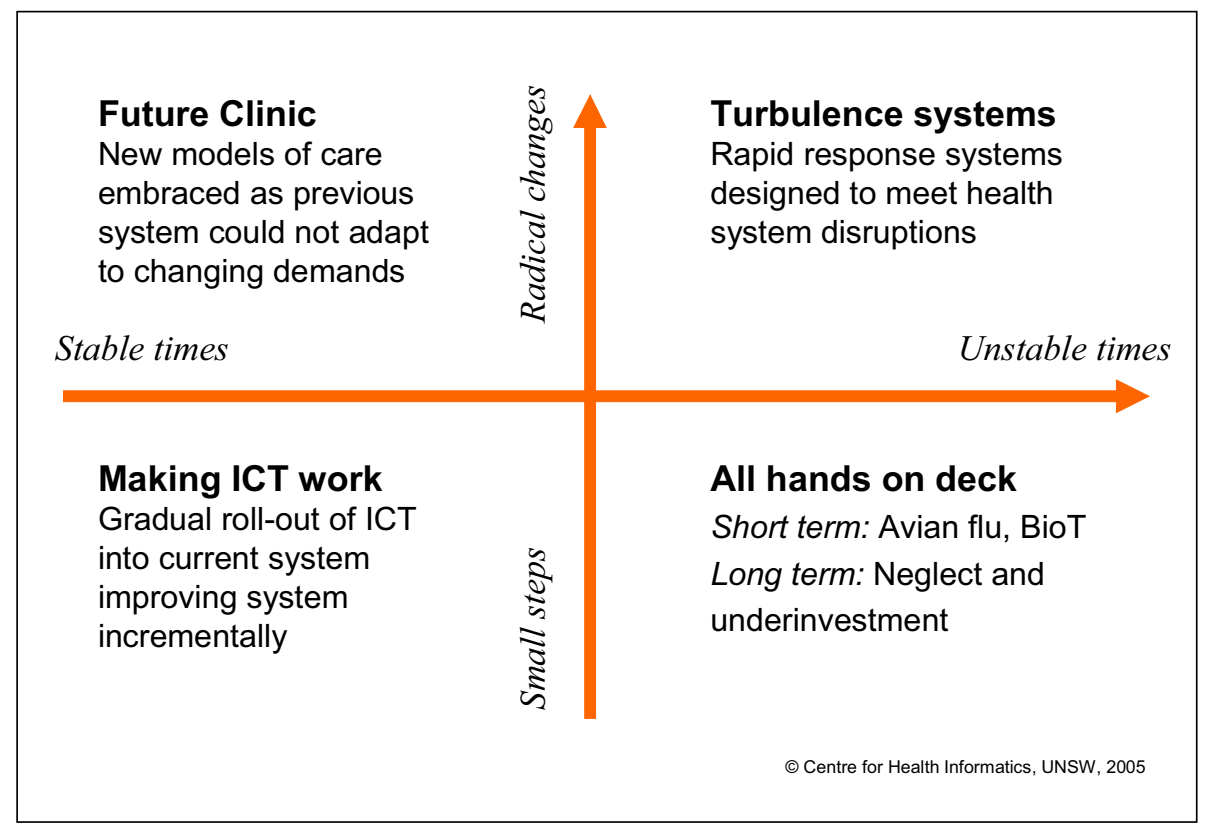

Fig. 1 CHI's research program is driven by a series of scenarios that describe different futures for the health system, and different responses from the research community to meet the needs of these different possible worlds might be done, and envisage ways of working that are only possible through the innovative use of ICT.

3. Turbulence systems: The risk of major shocks to the health system are ever present, including pandemics like avian flu, weather events of 'mass dimension' like Hurricane Katrina in New Orleans, and bioterror. It is possible to make preparations for these unstable times, and we envisage that there is a role for 'turbulence' management systems to assist health services detect shocks as early as possible, and manage them as best they can when they arrive. Detecting disease outbreaks as early as possible maximizes the health system's ability to mount reasonable responses, for example.

4. All hands on deck: In this scenario, health services receive major shocks in the short term, without the advantage of 'turbulence management' systems to assist in co-coordinating a response - for example a series of major weather events or a new global pandemic would all stretch the present health system in all countries beyond its capability to respond. Another road to this scenario in the long term is to not prepare for events like global warming or infectious disease outbreaks or an ageing population, and through underinvestment or poor planning, do nothing. We believe the best role researchers can play in such testing circumstances is to step aside from research and work directly within the health services in those places our skills and experience are most valuable. We look forward to not having to work in this quadrant.

Each of our research programs are shaped to meet needs in at least one of the first three quadrants, and we routinely revisit our scenarios to update our modeling of health system needs, and check to see that our programs are aligned with our best understanding of where the health system may head over the next few years. 


\section{A Problem-driven and Socio- technical Research Model}

Underpinning our approach to research is an understanding that health informatics is essentially an applied research discipline, and therefore that it must be strongly problem-driven. Consequently we see research as an iterative process of understanding the problem context and user needs within that context, designing systems or artefacts that meet those needs, and then evaluating our designs to make sure we have met the initial design specifications (Figure 2). Central to our research philosophy is also an understanding that we need to see any ICT design as a construct that works within a complex socio-technical environment [2]. We must therefore both ensure the design takes into account not just the cognitive needs of individual users, but also reflects the team environment within which individuals operate [3]. Social systems shape the way people react to and use technology [1], and generate events, pressures and resource limitations that alter, sometimes radically, the operating environment within which design must operate. There is a world of difference between a design that works in a laboratory setting with a single user, and the same design operating in a real world setting filled with interruptions, disturbances, competing tasks, and time and resource limitations.

To ensure we are able to meet the multi-disciplinary research challenges inherent in this research model, we have adopted a matrix-management approach to our research portfolio (Figure 3), where we have identified two main problem areas within which we will work - decision-making and communication, and a number of competencies needed to attack these problems. Thus our scenario planning generates a

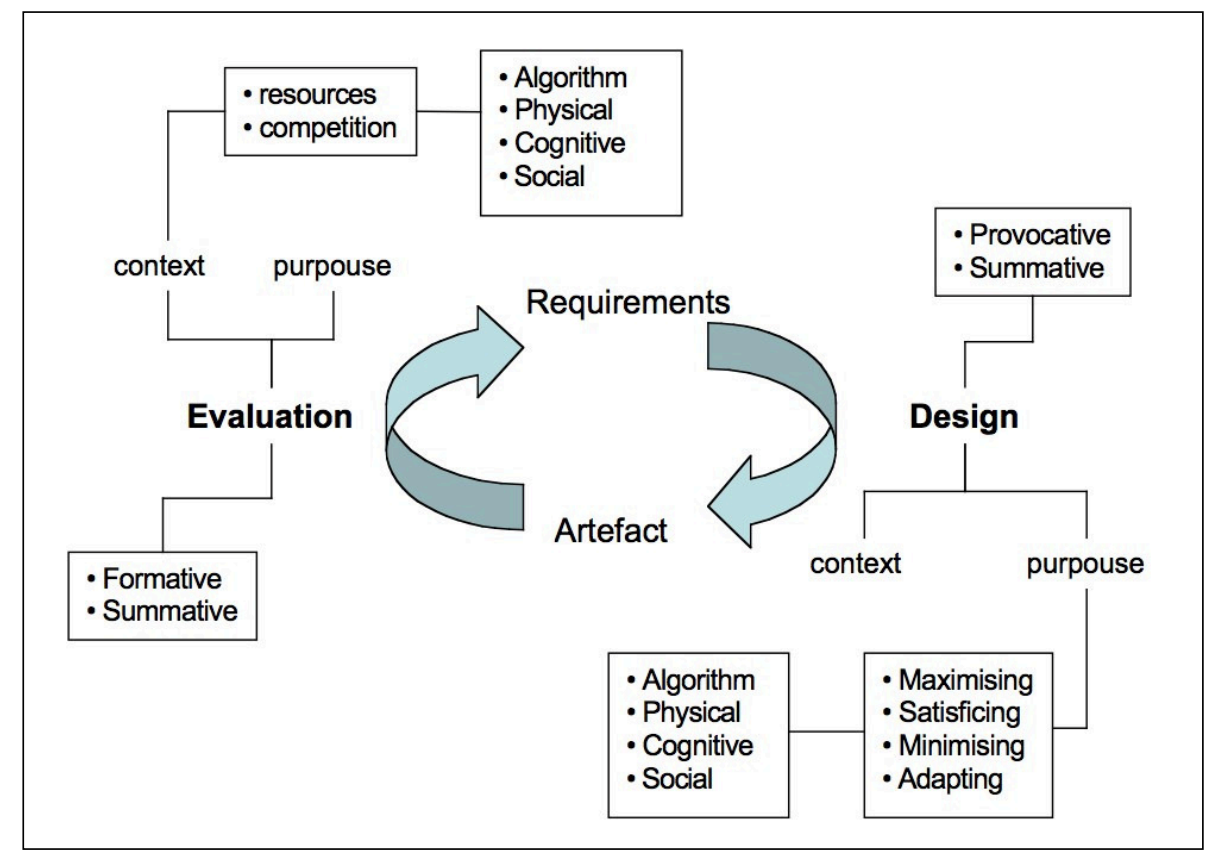

Fig. 2 Evaluation and Design shape the framing of requirements and artefacts, at each level of design from algorithmic description of task needs through to social setting (adapted from Coiera, 2007 (in press))

set of futures which drive the identification of problems we wish to tackle through sustained research effort, and the competency matrix is used to help us ensure we have developed capabilities in the research methods and tools we need to adequately and effectively address these problems. It is our goal to excel both in our ability to solve the challenges of these individual problem areas, as well as to excel in these areas of competency. Our domain of application for these two key problems of decision-making and communication extends from primary care physicians, through hospital settings, and more recently, the biomedical research workbench.

\section{The Research Programs}

\section{Communication}

If information is the lifeblood of healthcare, then communication is the heart that pumps it [4]. Yet communi- cation research is still in its infancy within the informatics research community. Researchers at $\mathrm{CHI}$ have over the last decade been widely recognized for their research into health care organization communication. With a goal to develop 'new ways' of working (scenario 2), we have contributed to highlighting the importance of this topic [5], developing theoretical models to help understand communication [6,7], developed observational methods to measure communication processes [8], and contributed basic research that specifically highlights the very high communication loads under which health care workers operate [9-12].

Traditionally health informatics research has focused on information system design, with an emphasis on the representation and storage of information, for example the health record. The support of communication has received little attention, despite the fact that up to $90 \%$ of the information transactions in some health services do not involve stored electronic data, but rather the 


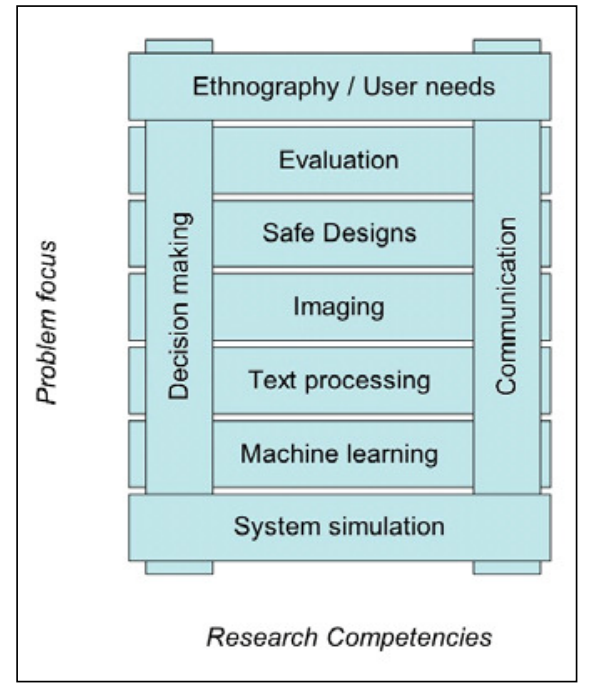

Fig. 3 Research program matrix. The Centre for Health informatics is focussed on working on problems in decision-making and in communication support, and on developing core competencies in some of the cross-problem methods needed to support these two broad problem areas

exchange of information between clinicians, often in face-to-face conversation [5]. However, the importance of supporting effective communication in health care is growing. Health care is not always safe or effective, and the role of poor communication in generating avoidable error and poor outcomes is now widely discussed. For example, we have argued that highly interruptive media such as the pager and the telephone, often the main means for communication between health professionals, can cause memory disruptions and lead to errors [7]. Since communication inefficiency and failure may be a core generator of clinical error [5, 7], supporting more effective communication practices may have a great impact on the quality and safety of health service delivery.

Health informatics has a key role in shaping our understanding of the role of communication in health care processes, and in crafting interventions to support improved communication. Information and communication technology seems to be a promising means for restructuring many communication processes, and there currently are an increasing array of communication channels, media, and devices from which communication services can be constructed. Typically research in communications is targeted at creating:

- Descriptions of the way communication processes are organized within health care, that can inspire the design and implementation of communication supporting systems;

- Demonstrations of the effects of the deployment of communication supporting applications on communication processes within health care. We are conducting an ongoing series of projects in both of these areas, looking at communication processes, their relationship to safety and clinical error, and looking to design new systems to support communication.

\section{Decision Support and Information Retrieval}

Supporting good decision-making by clinicians and consumers is a task that is central to all the future scenarios we have already described. The main focus of our work in this stream over the last decade has been to specifically support evidence-based decision making, and recognising that for most clinicians, the 'evidence' will still take the form of documents, whether they be published clinical practice guidelines, text books, or research papers published in the literature. Consequently we have focussed heavily on understanding the impact of information retrieval systems on clinical decision making, and on understanding how best to design such systems to maximise their use and clinical effectiveness, as measured by improved decision outcomes.

While present generation search technologies have made improvements to evidence accessibility, clinicians still have large unmet information needs. For example, doctors fail to find the information they need because they are unsure what is available, where to look for it, have very limited time available to conduct searches, and when they do search they have poor query formulation skills and often abandon searches because of time pressure. Often the evidence needed is scattered across heterogeneous data sources, each with their own unique query and indexing methods, creating further barriers.

A major outcome of our work has been the design and evaluation of an advanced knowledge-based information retrieval technology we call Quick Clinical (QC) [13]. The QC user model guides clinicians to first consider the purpose of their search through selection of a task specific search profile, and it then asks them to provide specific keywords related to that search task. As a consequence, users are guided through a process that structures their query for them and improves the chances that they will ask a wellformed query and receive an appropriate answer. Figure 4 depicts the QC search interface. On the left hand is a list of search filters that describe typical search tasks which are customized to the specific information needs of primary care physicians.

QC is driven internally by a rule-based mechanism to search only the most relevant of all the available resources, translating and enhancing user queries into the respective query languages of each resource. The underlying design concept is the notion of a Meta-search filter (MSF) which combines the power of meta-search systems and predefined search filter technologies [13]. They can be thought of as encodings of search strategies that capture expert knowledge on where and how to search for answers. A MSF might describe which 


\section{QuickClinical}

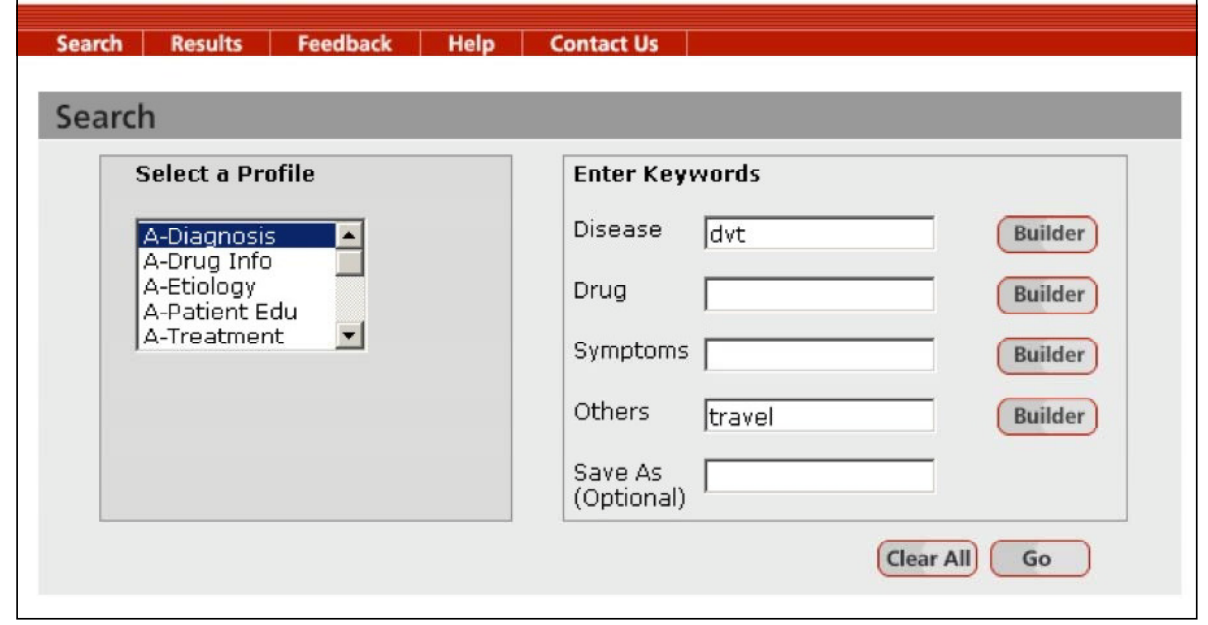

Fig. 4 Quick Clinical search screen

repositories are most appropriate in answering a typical question for researchers in a given discipline, and how best to ask the question within different resources. MSFs can be designed to support specific user groups, and different tasks and contexts associated with each group. For example, the strategy to search for information on the treatment of a disease for a researcher conducting clinical trials and for a researcher conducting basic science research in a laboratory would probably need to return very different documents sets, probably derived from different resources, reflecting the different skills and needs of these two groups.

QC has undergone multiple stringent evaluations between 2001-6, both in controlled laboratory settings, and in routine use in a primary care setting [14-17]. QC has had a demonstrated impact on the decision-making behaviors of primary care physicians, significantly improving the accuracy and timeliness of decision made. QC improves decision accuracy by $20 \%$ and using QC is much faster than standard on-line methods (on average 4.5 Vs $6.6 \mathrm{~min}$ utes), making it more likely to be used routinely. Estimating that 1 minute added to a primary care consultation in Australia would require an additional 800 physicians to provide the same service levels, saving 2 minutes per consultation represents a significant contribution to primary care service provision.

Apart from ongoing developments with search technologies, our research is now turning to related challenges. We are conducting analyses of the ways clinicians and consumers actually use the evidence they find with retrieval systems to make decisions. It seems that this form of decision making is as prone to traditional cognitive biases like the anchoring, primacy and recency effects as are other forms of decision making [18]. Consequently it should be possible to use this understanding of the cognitive processes used when analysing evidence to design better and safer retrieval systems, more likely to result in users finding the right evidence, and using that evidence to come to the right conclusion. We are also now working on the challenge of evidence summarisation. Whilst search systems continue to improve, the challenge of interpreting the many relevant docu- ments that are identified remains a challenge. Out text processing work aims to develop robust and valid means to summarise the content of multiple documents such as research papers, and provide clinicians with succinct and clinically relevant summaries of the current evidence.

\section{Public Health Surveillance}

The main focus for our work in the area of 'turbulence systems' is with emerging infectious diseases, and the pressing problem that current biothreat surveillance systems are vulnerable to incomplete and delayed reporting of such public health threats. Recent outbreaks of re-emerging and new communicable diseases have highlighted inefficiencies in public health monitoring and control systems. Our program of biosurveillance research is focused on informatics approaches to optimizing risk assessment and response to outbreaks of communicable diseases and addresses the need for improvement in the timeliness and specificity of early detection and control of biothreats.

New health indicator surveillance systems, including syndromic surveillance systems, are potentially more rapid and sensitive than traditional methods for detection of outbreaks or bioterrorismrelated events. They monitor health care utilization patterns, in real time, and rely on detecting case features that are discernable before laboratory diagnoses are confirmed. Reporting sources include emergency departments, laboratories, intensive care units, and hospital admission and discharge systems. However, these are surrogate markers and they may introduce confounding factors and noise into the outbreak 'signal'.

Improvement in the sensitivity and specificity of surveillance signals is impossible without some knowledge of 
disease-specific patterns that can be compared with daily counts from surveillance systems. Knowledge about specific infections with the potential for epidemics allows the formulation of probabilistic templates of syndromic surveillance signals, which can create epidemic curves. These templates can be used as filters to distil relevant information from the background noise in a population and trigger focused and rapid testing of patients with severe infection.

We have been developing new models of outbreak detection and risk assessment based on coordinated syndromic and laboratory diagnostic surveillance (Figure 5). Specific epidemic footprints for the main infectious disease syndromes are being developed and piloted in clinical setting as templates to trigger enhanced laboratory investigations. The low rate of actual outbreaks makes it difficult to calibrate such systems, much less validate disease specific templates. Consequently there is a need for in silico simulations of outbreaks using our best guesses about epidemic behaviour to tune the detection capability of detection systems.

Our work is also focused on one of the main barriers to the efficient monitoring of, and response to outbreaks, namely suboptimal and delayed decision-making, by providing new modes of decision support and integration of complex surveillance signals into action plans. Innovative analytic approaches using Bayesian classifiers and direct data based pattern recognition and clustering methods are applied to build rule-based decision support systems for clinical and public health assessments. This research also extends our current development of machine learning algorithms to provide patientspecific recommendations based on the molecular typing of bacteria with epidemic potential.

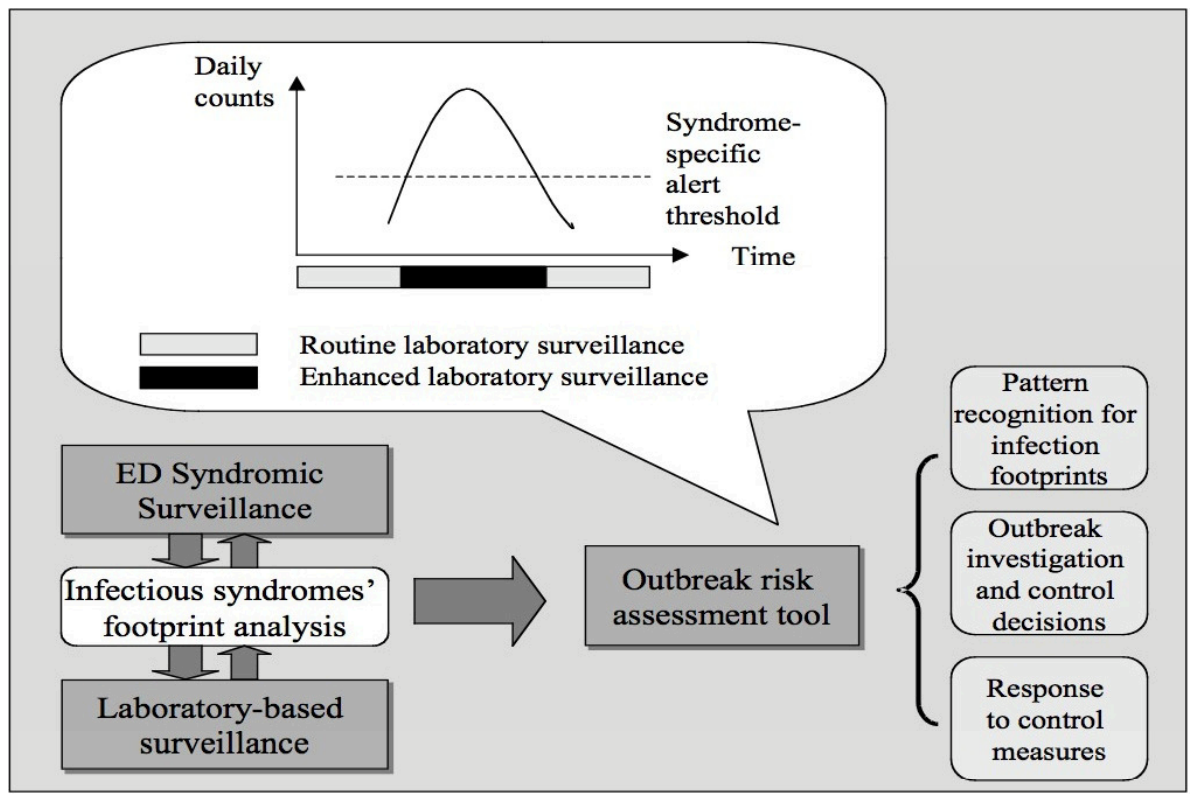

Fig. 5 Infectious disease outbreak detection and risk assessment based on coordinated syndromic and laboratory diagnostic surveillance.

\section{Safety and Evaluation}

System safety is critical to the success of the large-scale computerization being undertaken to improve the quality of health services delivery worldwide (Making ICT work, scenario 1). There is preliminary evidence that poorly implemented clinical decision support systems (CDSS) can lead to increased mortality in some settings [19]. Studies in the US, UK and Australia have found commercial prescribing systems often fail to uniformly detect significant drug interactions, probably because of errors in their knowledge bases. Electronic medication management systems may generate new types of error because of user-interface design, but also because of events in the workplace such as distraction affecting the actions of system users.

Another potential source of CDSS influenced errors are automation biases, including errors of omission where individuals miss important data because the system does not prompt them to notice them, and errors of commission where individuals do what the decision aid tells to do, even when this contradicts their training and other available data. Errors of dismissal occur when relevant alerts are ignored. On-line decision support systems may also result in errors where clinicians come to an incorrect assessment of the evidence, possibly shaped in part by cognitive decision biases [15].

Our safety research program is focusing on understanding the ways in which ICT is both an enabler of safer clinical practice, and also understanding the ways in which ICT can unintentionally be the generator of new types of error [20]. Our research aims to use this rich understanding of the way ICT impacts safety to develop innovative tools and methods to improve the safety of clinical information systems. The specific focus of the program is clinical decision support [19], including an examination of the medication management and prescribing processes to ensure that the outcomes of this research will have immediate impact on patient safety in routine care [21]. We also wish to influence policy in this area, and begin a 
debate about the best mechanisms to ensure that clinical software is safe, and does not inadvertently result in patient harm or misadventure [22].

\section{Imaging Informatics}

Medical imaging research aims to develop systems that make efficient use of information and communication technology to facilitate the radiological interpretation process. The number of images available to radiologists is growing rapidly and has outpaced the human ability to process them. Computational aids are required to filter the large number of images now produced by individual patient studies, and to focus the radiologist's attention on diagnostically interesting features in an image set.

Our research in image informatics aims to improve image-based disease detection and diagnosis and monitoring of treatment outcomes. We are investigating novel methods for automating the process of image interpretation, combining knowledge based and modelbased approaches to improve the processing and the analysis of medical images. We are making extensive use of machine learning to acquire rules for detecting the presence of various diseases patterns.

In addition to image analysis and interpretation we are actively involved in the development of tools for modeling and $3 \mathrm{D}$ visualization of medical data. The results of image analysis are best presented in the form of a 3D model of the imaged anatomy that can be manipulated interactively. With a wellequipped laboratory for 3D scientific visualization, each year we attract undergraduate and postgraduate computer science and biomedical engineering students to work with us. Current research projects include:

- Computer Aided Detection and Di- agnosis of Diffuse Lung diseases [23-25]

- 3D Modeling and Visualization of the lung [26, 27]

- Anatomical atlases [28]

- Interactive Radiology Forum, a teleradiology system that enables a real time conference multimedia chat facility to exchange expert opinion on images from radiology practice.

- A Web-based system for an Efficient Medical Image Information Retrieval from large multimedia medical data repositories [29]

- A system for extracting, interpreting and structuring information from clinical free text reports in a form to facilitate decision support and diagnosis

- Techniques for efficient integration and 3D visualization of data from heterogeneous sources to assist with the geo-spatial mapping and early warning of infectious disease outbreaks

\section{Biomedical Systems Simulation}

Simulation is a core competence for our Centre, and is used to support a wide variety of projects, across all research streams and problem domains. Health Systems Simulation is the application of modeling and computer simulation methods to explore, understand and improve the interaction between structure and action in health care and policy [30]. Simulation provides dynamic analysis of the health system, predicting how the health system might behave over time. Simulation can support, replace or extend upon health service studies such as randomized controlled trials when they are otherwise impossible, too time-consuming, or too expensive to undertake in the real world. Since the health system is a multi-scale system, our approach is to use a combination of modeling methods to depict both the aggregate and disaggregate view of the system. System dynamics models are used to outline the context of the problem, making explicit the boundaries of the health system, its structure and relationships. Using this approach we can identify reinforcing and balancing feedback effects, circular causation, delayed responses and the leverage points where decisions and actions bring about changes to the systems performance. Combining this with agent based modeling, we can capture emergent and non-linear behavior arising from the interactions at the individual level and how the system learns, adapts and self-organizes itself to maintain order.

Using these modeling and simulation methods, we are able to develop hypotheses about the structure of health services (components and their interactions), from the detail level to the overall context, that is both necessary and sufficient to account for the specific behavior of interest or health system problem. The simulation tests this hypothesis by enabling comparisons with data and observed behavior with the derived behavior. Behavior modes of interest include overshoot and collapse or damped oscillation with a certain period (for example, health workforce over- and under-supply cycles). This is valuable whenever decision makers misperceive the system and draw wrong conclusions about behavior and therefore about the choice of policies. Current simulation projects are examining the safety of current prescribing and medication management [21] and the design of safer approaches to electronic medication management, modeling the impact of ICT on clinical work, and the impact of performance measures on the behavior of health service managers.

We are also exploring general-purpose methods of building complex models from multiple sub models (multi modeling) where the smaller models may 
be of many different representations and scales. To this end, we have developed a Field Representation Language (FRL) which aims to provide a scale-independent, method-independent representation of spatio-temporal information, using the mathematical notion of a field as the integrating representational concept [31]. FRL has been used to integrate a stochastic model of the deposition of micro-particles throughout the microvasculature of a tumour, with a finite-element model of ferromagnetic embolisation hyperthermia [32].

\section{Conclusion}

The health and biomedical informatics research sector will underpin many of the innovations needed to develop truly sustainable health care services over the next decades, and its impact will be felt both in clinical practice, as well as in the research sector. Any individual research group like CHI must necessarily focus on a few areas to allow it to develop sufficient research capacity to make novel and internationally significant contributions. As CHI approaches the end of its first decade, it is becoming clear that developing capacity becomes increasingly challenging as the research territory changes under our feet. New methods, new opportunities from innovations such as biobanks, large scale clinical data sets, micro array assays and the like ensure that the research programs described here are but a snapshot in time, and that the Centre will continue to evolve and shift its focus in the years to come.

\section{References}

1. Coiera E. Four rules for the reinvention of healthcare. BMJ 2004;328:1197-9.

2. Coiera E. Getting technical about socio-technical systems science. Int J Med Inform 2007;in press.

3. Coiera E. Interaction design theory. Int $J$ Med Inform 2003;69:205-22.

4. Toussaint PJ, Coiera E. Supporting communication in health care. Int J Med Inform 2005;74(10):779-81.

5. Coiera E. When conversation is better than computation. JAm Med Inform Assoc 2000;7(3):277-86.

6. Coiera E. Mediated agent interaction. In: Quaglini $\mathrm{BaA}$, editor. 8th Conference on Artificial Intelligence in Medicine. Berlin: Springer Lecture Notes in Artificial Intelligence No. 2101; 2001.

7. Parker J, Coiera E. Improving Clinical Communication: a view from Psychology. $J \mathrm{Am}$ Med Inform Assoc 2000; 7:453-61.

8. Spencer R, Logan P, E C. Communication Observation Method (COM) Manual. Centre for Health Informatics, UNSW; 2002.

9. Coiera E, Tombs V. Communication behaviours in a hospital setting: an observational study. $\mathrm{Br} \mathrm{Med} J$ 1998;316:673-7.

10. Coiera E, Jayasuriya R, Hardy J, Bannan A, Thorpe M. Communication loads on clinicians in the emergency department. Med J Aust 2002;176:415-8.

11. Alvarez G, Coiera E. Interruptive communication patterns in the intensive care unit ward round. Int $J$ Med Inform 2005;74:791-6.

12. Spencer R, Coiera E, Logan P. Variation in communication loads on clinical staff in the emergency department. Ann Emerg Med 2004:44:268-73.

13. Coiera E, Walther M, Nguyen K, NH L. An architecture for knowledge-based and federated search of online clinical evidence. $J$ Med Internet Res 2005 Oct 24;7(5):e52.

14. Magrabi F, Coiera EW, Westbrook JI, Gosling AS, Vickland V. General practitioners' use of online evidence during consultations. Int J Med Inform 2005;74(1):1-12.

15. Westbrook J, Coiera E, Gosling AS. Do online information retrieval systems help experienced clinicians answer clinical questions? JAm Med Inform Assoc 2005;12(3):315-21.

16. Magrabi F, Westbrook JI, Coiera EW, Gosling AS. Clinicians' assessments of the usefulness of online evidence to answer clinical questions. Medinfo 2004:11(pt 1):297-300.

17. Westbrook J, Gosling A, Coiera E. The impact of an online evidence system on confidence in decision making in a controlled setting. Med Decis Making 2005;25:178-85.

18. Lau A, Coiera E. A Bayesian model that predicts the impact of Web searching on decision-making. $J$ Am Soc Inf Sci Technol 2006;57(7):873-80.

19. Coiera E, Westbrook JI, Wyatt JC. The Safety and Quality of Decision Support Systems. Methods Inf Med 2006;45(1):S20-5

20. Ash J, Berg M, Coiera E. Some unintended consequences of information technology in health care: The nature of patient care information systems related errors. J Am Med Inform Assoc 2004;11(2):104-12.

21. Toussaint P, Williamson M, McDonnell G, Nytro $\varnothing$. Modeling the Communications Dimension of Clinical Work and Medication Errors. The 24th International Conference of the System Dynamics Society. Nijmegen, The Netherlands; 2006.

22. Coiera EW, Westbrook JI. Should clinical software be regulated? Med JAust 2006;184(12):600-1.

23. Wong J, Zrimec T. Classification of Lung Disease Pattern Using Seeded Region Growing,. AI 2006: Advances in Artificial Intelligence. Hobart, Australia: Springer. 2006. p. 233-42.

24. Wong J, Zrimec T. Automatic Honeycombing Detection Using Texture and Structure Analysis, . ICSC Congress on Computational Intelligence: Methods \& Applications ; 2005.

25. Busayarat S, Zrimec T. Automatic Detection of Pulmonary Arteries and Assessment of Bronchial Dilatation in HRCT Images of the Lungs. ICSC Congress on Computational Intelligence: Methods \& Applications, 2005.

26. Busayarat S, Zrimec T. Ray-Tracing Based Registration for HRCT Images of the Lungs. Medical Image Computing and Computer-Assisted Intervention-MICCAI; 2006. p. 670-7.

27. Busayarat S, Zrimec T P W. Knowledge-Directed Automatic Bronchi Detection Method for Sparse HRCT Scans of the Lungs. Image and Vision Computing. New Zealand; 2005. p. 369-74.

28. Zrimec T, Busayarat S, Wilson P. A 3D Model of the Human Lung. Medical Image Computing and Computer-Assisted Intervention-MICCAI 2004. Berlin: Springer; 2004. p. 1074-5.

29. Zrimec T. A Content Based Retrieval System for Medical Images. The Seventh International Conference on Control, Automation, Robotics and Vision, ICRACV-2002. Singapore; 2002.

30. McDonnell G, Heffernan M, Faulkner A. Using System Dynamics to analyse Health System Performance within the WHO Framework. 22nd International Conference of the System Dynamics Society; 2004. p. 25-9.

31. Tsafnat G, Cloherty S L, Lambert TD. AFL and FRL: Abstraction and representation for field interchange. The 26th Annual Conference on Biomedical Engineering. IEEE Engineering in Medicine and Biology Society: Omnipress; 2004.

32. Tsafnat N, Tsafnat G, Lambert TD, Jones SK. Modelling heating of liver tumours with heterogeneous magnetic microsphere deposition. IOP Journal of Physics and Biology 2005;50 (12):2937-53.

\section{Correspondence to:}

Enrico Coiera

Centre for Health Informatics

University of New South Wales

UNSW 2052 NSW Australia

Tel: +61 293859026

Fax: +61 293859006

E-mail: e.coiera@unsw.edu.au 